\title{
The Study on the Effect and Mechanism of Crocin against Human Ewing Sarcoma
}

\author{
DAWEI WANG ${ }^{1}$, YINGSHUANG WU'1, QINGHUI JI2, YONGXIANG LIU³ ${ }^{3}$ YI LUO'1 AND JINGLONG YAN*
}

Department of Orthopedics, The Second Affiliated Hospital of Harbin Medical University, Harbin 150000, ${ }^{1}$ Department of Orthopedics, Zhangjiakou First Hospital, Zhangjiakou 075000, ${ }^{2}$ Department of Orthopedics, The First Affiliated Hospital of Jiamusi University, Jiamusi 154000, ${ }^{3}$ Department of Orthopedics, Yantaishan Hospital, Yantai 264000, China

\section{Wang et al.: Effect and Mechanism of Crocin against Human Ewing Sarcoma}

\begin{abstract}
Ewing sarcoma is the second most common primary malignant bone tumor in pediatrics after osteosarcoma, which mainly occurs in children and adolescents aged 5-20 y. Crocin is the main active ingredient extracted from Crocus sativus and has significant inhibitory effect on many cancer cells. Therefore, this study observed the effect of crocin on human Ewing sarcoma and its specific mechanism and provided molecular theoretical basis for clinical application. We cultured Human Ewing sarcoma cells lines SKES-1 and RD-ES in vitro and treated them with $0,2.5,5$ and $10 \mathrm{mmol} / \mathrm{l}$ crocin solution. And we used cell counting kit-8 assay to detect cell viability and adopted flow cytometry to detect cell apoptosis and cell cycle changes, used western-blot to detect the expression of apoptotic pathway-related proteins. At the same time, we also combined vincristine and crocin to treat the cells, to detect cell viability and apoptosis, and to observe for synergistic effect. We established the xenografts model of Ewing's sarcoma in nude mice and observe the inhibitory effect of crocin and vincristine on xenografts. Crocin could not only inhibit the growth of human Ewing sarcoma cells SK-ES-1 and RD-ES in a dose-dependent manner, but also inhibit the growth of xenografts in nude mice and increase cell apoptosis and block cell cycle progression. The results of mechanism analysis showed that crocin and vincristine significantly increased the degradation of poly adenosine diphosphate-ribose polymerase and caspase-3, and increased the levels of anti-apoptotic proteins B-cell lymphoma 2, B-cell lymphoma-extra-large and p21. And crocin could increase the effect of vincristine on proliferation inhibition and apoptosis increase of human Ewing sarcoma. Crocin could inhibit the growth of human Ewing sarcoma either alone or in combination with vincristine. This effect was mediated by increasing apoptosis-related pathways.
\end{abstract}

Key words: Ewing sarcoma, crocin, cell survival, vincristine, synergistic effect

Ewing sarcoma ${ }^{[1]}$ is the second most common primary malignant bone tumor in pediatrics after osteosarcoma, it mainly occurs in adolescents aged 5-20 y and has a high degree of clinical malignancy. Nearly $30 \%$ of the patients have occurred metastasis in other parts of the lung and bone at the time of diagnosis. Surgical resection is often difficult to cure and is prone to appear recurrence and metastasis, and the prognosis is very poor. In recent years, with the continuous progress in neoadjuvant chemotherapy, radiotherapy and surgery, has effectively improve the treatment effect of patients, however the 5 y survival rate is still less than $50 \%$. At present, the continuous development of multi-modality therapy, mainly chemotherapy, radiotherapy and surgery, has greatly increased the long-term survival rate of primary tumors, while the effect is still not satisfactory if the patient with distant metastasis. And giving high-dose intensive chemotherapy will cause serious side effects such as bone marrow suppression, gastrointestinal reaction, cardiotoxicity, hepatotoxicity, kidney and bladder toxicity etc., which are difficult to tolerate. At the same time, if the patient has a small metastasis at the time of diagnosis, the treatment effect is worse. Therefore, it is of great clinical significance to find effective treatment methods. 


\section{MATERIALS AND METHODS}

Human Ewing sarcoma cell lines SK-ES-1 and RD-ES (American Type Culture Collection (ATCC), USA) were cultured in Roswell Park Memorial Institute Medium (RPMI)-1640 medium containing $10 \%$ fetal bovine serum (FBS) in incubator at $37^{\circ}$ and $5 \%$ Carbon Dioxide $\left(\mathrm{CO}_{2}\right)$, the cells were digested with Ethylene Diamine Tetra-acetic Acid (EDTA) trypsin and subcultured in cell culture flask. Cell cycle and apoptosis detection kit (China Nantong Biyuntian Biotechnology Research Institute, Nantong, China). Enhanced Luminol-Based Chemiluminescent (ECL) Immunoblotting Substrate Kit (Millipore, USA). Caspase-3 and Poly Adenosine Diphosphate-Ribose Polymerase (PARP) antibodies (Abcam, USA). Flow cytometry used FACS Aria II, microplate reader (Bio-Rad, USA) ${ }^{[2]}$.

\section{Culturing and grouping of cells:}

Put the SK-ES-1 and RD-ES cells in $90 \%$ RPMI1640 medium containing $10 \% \mathrm{FBS}$ in an incubator at $37^{\circ}$ and $5 \% \mathrm{CO}_{2}$ and $90 \%$ saturated humidity for culturing, which should be noted that the solution should be changed every other day. When $80 \%$ of the cells were fused, used $0.25 \%$ trypsin-EDTA for cell digesting and passage, using logarithmic growth phase cells in all experiments. Cells were divided into four groups: $0 \mathrm{mM}$ group: cells were not treated; $2.5 \mathrm{~mm}$ group: crocin at $2.5 \mathrm{mmol} / 1$ concentration, $5 \mathrm{~mm}$ group: crocin at $5 \mathrm{mmol} / \mathrm{l}$ concentration, $10 \mathrm{mM}$ group: crocin at $10 \mathrm{mmol} / 1$ concentration. When the mechanism of apoptosis and the synergistic detection of vincristine were carried out, the cells were treated with crocin at the concentration of $10 \mathrm{mmol} / \mathrm{l}$ in the positive drug group and vincristine at the concentration of $1 \mathrm{ng} / \mathrm{ml}$.

\section{Preparation of crocin solution:}

Dissolved $1000 \mathrm{mg}$ crocin in $20 \mathrm{ml}$ Phosphate Buffered Saline (PBS) solution $(0.01 \mathrm{~mol} / \mathrm{l}, \mathrm{pH}=7.2)$ and 62.5 mg EDTA (crocin stabilizer) was added to prepare 50 $\mathrm{mg} / \mathrm{ml}(50 \mathrm{mmol} / \mathrm{l})$ storage solution, which was stored at $4^{\circ}$. PBS $(0.01 \mathrm{~mol} / \mathrm{l}, \mathrm{pH}=7.2)$ was diluted as the appropriate concentration for clinical use; and 0, 2.5, 5 and $10 \mathrm{mmol} / \mathrm{l}$ were selected as the experimental concentration of crocin.

\section{Detection of cell proliferation by Cell Counting Kit- 8 (CCK-8) method:}

Adjusted the cell concentration to $3 \times 10^{4}$ cells $/ \mathrm{ml}$ and inoculated in 96-well plate with 100 holes per hole. Discarded the supernatant after $24 \mathrm{~h}$ of incubation. Added crocin $(0,2.5,5,10 \mathrm{mmol} / \mathrm{l})$ of different concentrations into each hole. Six compound holes were set up in each group and the cells were incubated at $37^{\circ}, 5 \% \mathrm{CO}_{2}$ incubator for $24 \mathrm{~h}$ and $48 \mathrm{~h}$ respectively, each hole was incubated with $10 \mu \mathrm{l}$ CCK-8 solution, after incubation at $37^{\circ}$ for $2 \mathrm{~h}$, the absorbance (A) value was read by an automatic enzyme-labeled plate reader at a wavelength of $450 \mathrm{~nm}$ and $650 \mathrm{~nm}$ was set as the reference wavelength, the cell proliferation rate was calculated by deducting the reference A value.

\section{Detection of cell cycle changes by flow cytometry:}

Adjusted the cell concentration to $3 \times 10^{4}$ cells $/ \mathrm{ml}$ and inoculated in 96-well plate with 100 holes per hole. Discarded the supernatant after 24 h of incubation. Added crocin $(0.5 \mathrm{mmol} / \mathrm{l}) 100 \mu \mathrm{l}$ of different concentration into each hole. Six multiple holes were set up in each group, after $24 \mathrm{~h}$ of incubation at $37^{\circ}$ and $5 \% \mathrm{CO}_{2}$, the cells were cleaned by pre-cooled PBS solution for 2 times, $100 \mu \mathrm{l}$ PBS were added to re-suspend the cells and after added pre-cooled ethanol $400 \mathrm{ml}$ fixing for $1 \mathrm{~h}$, removed the ethanol by centrifugation, the cells were cleaned by pre-cooled PBS solution for 2 times, $100 \mathrm{ml}$ PBS suspended cells were added again, $50 \mu \mathrm{g} /$ $\mathrm{ml}$ Propidium Iodide (PI) was added and the cells were stained for $30 \mathrm{~min}$ at $4^{\circ}$ and filtered by $400 \mu \mathrm{PBS}$, detected the cell cycle by flow cytometry at $480 \mathrm{~nm}$.

\section{Detection of apoptotic rate by flow cytometry:}

$100 \mu \mathrm{l}$ cells were incubated overnight at $37^{\circ}$ with $5 \times 10^{6}$ cells $/ \mathrm{ml}$ concentration, then different concentrations of drugs were given to intervene and the cells were incubated at $37^{\circ}$ for $24 \mathrm{~h}$, the cells were digested and collected, PI/annexin V-FITC was stained and used flow cytometry to detect apoptotic rate.

\section{Detection of caspase-3 and PARP protein expression by western blotting:}

After separating the total protein of the above different cells, the concentration of the cell protein was measured by Bicinchoninic Acid (BCA) method and then analyzed by Sodium Dodecyl Sulfate Polyacrylamide Gel Electrophoresis (SDS-PAGE) and transferred to a Polyvinylidene Difluoride (PVDF) membrane. After blocking the PVDF membrane with $5 \%$ skim milk, added the corresponding primary antibody, incubated at $4^{\circ}$ overnight, after using Tris-Buffered Saline with Tween 20 (TBST) washing the membrane for 3 times, added the corresponding secondary antibody, incubated for $2 \mathrm{~h}$ at room temperature, washed the membrane 3 times with TBST, performed color development by chemiluminescence kit, the protein bands obtained were quantified using Image $\mathbf{J}$ software and analyzed for protein levels. 


\section{Detection of anti-apoptosis expression by western blotting:}

After detecting the total protein of the cells, the protein levels of the anti-apoptosis-related proteins B-Cell Lymphoma 2 (Bcl-2), B-Cell Lymphoma-Extra Large (Bcl-xL) and p21 were detected according to the above experimental methods.

\section{Synergistic detection of crocin traditional chemotherapeutic drugs:}

The cells were treated according to the above experimental methods then inoculated into 96-well plates; we divided the cells into control group, crocin group (with the concentration of $10 \mathrm{mmol} / \mathrm{l}$ ), vincristine group (with the concentration of $1 \mathrm{ng} / \mathrm{ml}$ ), crocin group (with the concentration of $10 \mathrm{mmol} / \mathrm{l}$ ) and vincristine group (with the concentration of $1 \mathrm{ng} / \mathrm{ml}$ ). Each group was treated with 6 compound holes and then cultured in $37^{\circ}$ and $5 \% \mathrm{CO}_{2}$ incubator for $48 \mathrm{~h}$, we adopted the CCK-8 method to detect the cell proliferation rate and western-blotting method was used to detect the protein levels of caspase-3, PARP, bcl-2, Bcl-xL and p2 $1^{[3-5]}$.

\section{Establishment of subcutaneous implantation tumor model in nude mice and drug intervention:}

55 nude mice were given subcutaneous injection of $0.1 \mathrm{ml} \mathrm{SK-ES-1}$ cell suspension on the right back after $1 \mathrm{w}$ of adaptive feeding and observed the growth of xenografts daily. The formation of subcutaneous nodules $(>5 \mathrm{~mm}) 10 \mathrm{~d}$ later indicated that the model was established successfully and 51 nude mice were modeling successfully. We randomly divided them into crocin group, vincristine group, combined drug group and control group. Crocin group ${ }^{[6]}$ and vincristine group were intraperitoneally injected with $10 \mathrm{mmol} / 1$ crocin and $1 \mathrm{ng} / \mathrm{ml}$ vincristine respectively every day. The combined drug group was injected with two drugs at the same time. The control group was injected with PBS buffer solution for $5 \mathrm{~d}$. The length (a) and short diameter (b) of tumors were measured every $3 \mathrm{~d}$ by vernier caliper and the volume of tumors was calculated ${ }^{[7]}$.

\section{Detection of apoptosis-related proteins expression in xenografts by western blotting:}

After $5 \mathrm{~d}$ of continuous administration, we selected 6 nude mice randomly from each group, conducted resection of tumor tissue under chloral hydrate anesthesia, the expression of caspase-3, PARP, bcl-2, Bcl-xL and p21 in xenografts were detected by westernblotting.
All the data were expressed as "mean+Standard Deviation (SD)", the differences between the two groups and the other groups were compared by using paired $t$ test (double tail) and two-way Analysis of Variance (ANOVA) method, respectively. $p<0.05$ was considered to have statistical significance. All statistical methods were analyzed by GraphPad Prism 6.0 and Statistical Package for the Social Sciences (SPSS) 18.0 statistical software.

\section{RESULTS AND DISCUSSION}

In order to clarify the effect of crocin on Ewing sarcoma, we incubated different concentrations of crocin and Ewing sarcoma cell lines SK-ES-1 and RD-ES to detect cell proliferation by CCK- 8 method. Our results were shown in fig. 1, crocin could inhibit the growth of human Ewing's sarcoma cells SK-ES-1 and RD-ES in a dose-dependent manner; and at concentrations of $2.5,5$, and $10 \mathrm{mmol} / 1$ for $24 \mathrm{~h}$, there was a significant difference in cell growth from $48 \mathrm{~h}(\mathrm{p}<0.05)$.

In order to further clarify the effect of crocin on Ewing sarcoma, we investigated the changes of cell cycle by flow cytometry after incubation of different concentrations of crocin and Ewing sarcoma cell lines SK-ES-1 and RD-ES. Our results were shown in fig. 2, from the results we can see that crocin inhibited human Ewing sarcoma cells from the G1 phase to the S phase in a dose-dependent manner, blocking the cell cycle progression. At the concentrations of $2.5,5$ and 10 $\mathrm{mmol} / \mathrm{l}$, the Gap Phase/Growth Phase (G0/G1) ratio of the cells was significantly higher than that of the control group,and the difference was statistically significant. [(SK-ES-1: $(61.09 \pm 0.81) \%$ vs. $(68.13 \pm 0.79) \% v s$. (72.13 \pm 1.33$)$ vs. $(44.29 \pm 0.57) \%$; RD-ES: $(60.23 \pm 0.65)$ $\%$ vs. $(65.13 \pm 0.56) \%$ vs. $(74.21 \pm 1.76)$ vs. $(41.44 \pm 0.66)$ $\%]($ all $\mathrm{p}<0.05)$.

In order to further clarify the effect of crocin on apoptosis of Ewing sarcoma cells, we incubated different concentrations of crocin and Ewing sarcoma cell lines SK-ES-1 and RD-ES to detect apoptosis rate by Annexin V-PI staining. The results were shown in fig. 3. From the results can be seen that, crocin enhanced the apoptosis of human Ewing sarcoma cells SK-ES-1 and RD-ES in a dose-dependent manner; and at the concentrations of $2.5,5$ and $10 \mathrm{mmol} / \mathrm{l}$, the apoptotic rate of cells was significantly different from that of the control group [SK-ES-1: $15.2 \pm 2.4 \%$ vs. $25.2 \pm 3.8 \%$ vs. $35.2 \pm 4.6 \%$ vs. $6.2 \pm 1.2 \%$; RD-ES: $17.2 \pm 2.5 \%$ vs. $26.2 \pm 3.6 \%$ vs. $37.3 \pm 4.7 \%$ vs. $5.6 \pm 0.9 \%](\mathrm{p}<0.05)$. 
In order to further clarify the mechanism of crocin's effect on Ewing sarcoma, the effect of crocin at 10 $\mathrm{mmol} / \mathrm{l}$ on apoptotic molecules in Ewing sarcoma cell lines SK-ES-1 and RD-ES was detected by western blotting. Our results were shown in fig. 4 . It can be seen from the results that crocin could significantly increase the levels of PARP and caspase-3 with the prolongation of action time, thus promoting the apoptosis of Ewing sarcoma cells $(\mathrm{p}<0.05)$.

In order to further clarify the mechanism of crocin's effect on Ewing sarcoma; we used western blotting method to detect the effect of crocin at $10 \mathrm{mmol} / \mathrm{l}$ on anti-apoptotic molecules in Ewing sarcoma cell lines SK-ES-1 and RD-ES. Our results were shown in fig. 5. It can be seen from the results that crocin can significantly reduce the expression levels of bcl-2, Bcl$\mathrm{xL}$ and $\mathrm{p} 21$ proteins with prolonged action time, thus promoting the initiation of apoptosis in Ewing sarcoma cells $(\mathrm{p}<0.05)$.

In order to further clarify the synergistic effect of crocin and conventional chemotherapeutic drugs on Ewing sarcoma, we incubated Ewing sarcoma cell lines SK-ES-1 and RD-ES with crocin of $10 \mathrm{mmol} / \mathrm{l}$ and vincristine of $1 \mathrm{ng} / \mathrm{ml}$ and detected the cell viability by CCK-8 method. Results as shown in fig. 6 , the cell survival rates of Ewing sarcoma SK-ES-1 and RDES cells treated with $10 \mathrm{mmol} / 1 \mathrm{crocin}$ and $1 \mathrm{ng} / \mathrm{ml}$ vincristine were significantly lower than those treated with vincristine alone and crocin alone $(\mathrm{p}<0.05)$. [SKES-1: $64.8+4.6 \%$ vs. $65.7+3.8 \%$ vs. $24.9+6.8 \%$; RD-ES: $62.7+4.7 \%$ vs. $64.7+3.9 \%$ vs. $20.6+5.7 \%$ ], suggested that the combination of crocin and vincristine enhanced the inhibitory effect on the proliferation of Ewing sarcoma cells.

In order to further clarify the effect of crocin and conventional chemotherapeutic drugs on the apoptosis of Ewing sarcoma cells, we incubated Ewings arcoma cell lines SK-ES-1 and RD-ES with crocin of 10 $\mathrm{mmol} / \mathrm{l}$ and vincristine of $1 \mathrm{ng} / \mathrm{ml}$ and detected the expression of apoptotic protein and anti-apoptotic related protein by western blotting. Results as shown in fig. 7, crocin in Ewing sarcoma SK-ES-1 and RDES could significantly increase the levels of PARP and caspase- 3 and significantly decrease the levels of bcl-2, bcl-xL 2 and p21 proteins when crocin at $10 \mathrm{mmol} / 1$ was combined with $1 \mathrm{ng} / \mathrm{ml}$ vincristine $(\mathrm{p}<0.05)$. This suggested that the combined action of crocin and vincristine can promote the apoptosis of Ewing sarcoma cells and both of them have synergistic effect.

In order to further clarify the inhibitory effect of crocin on Ewing sarcoma, we successfully established SKES-1 cell xenograft model in nude mice and observed the inhibitory effect of crocin on xenograft in nude mice ${ }^{[8,9]}$. Results as shown in fig. 8, crocin could significantly inhibit the growth of xenograft tumors in nude mice, its effect was similar to that of $1 \mathrm{ng} / \mathrm{ml}$ vincristine, while the volume of tumors in combination group was significantly lower than that in crocin group and vincristine group, which suggested that the combination of crocin and vincristine could inhibit the growth of Ewing sarcoma.

The results were shown in fig. 9, when $10 \mathrm{mmol} / 1$ crocin combined with $1 \mathrm{ng} / \mathrm{ml}$ vincristine, the levels of PARP and caspase-3 in SK-ES-1 xenografts were significantly increased, while the levels of bcl-2, Bcl-xL and $\mathrm{p} 21$ protein were significantly decreased $(\mathrm{p}<0.05)$. It is suggested that crocin can promote apoptosis of SK-ES-1 xenograft tumor cells in nude mice and the combined effect of crocin and vincristine was stronger, both of them had synergistic effect ${ }^{[10]}$.

A

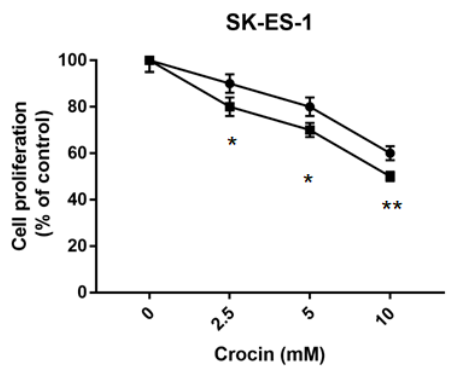

B

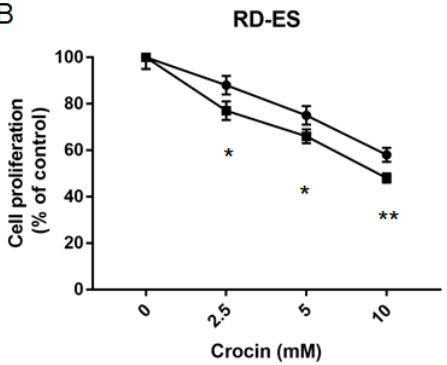

Fig. 1: Crocin inhibited the growth of human Ewing sarcoma cells, (A) SK-ES-1 and (B) RD-ES in a dose-dependent manner Note: *p<0.05 24 h vs. 48 h; **p<0.01 24 h vs. 48 h, (- ) 24h; (- ) 48h 

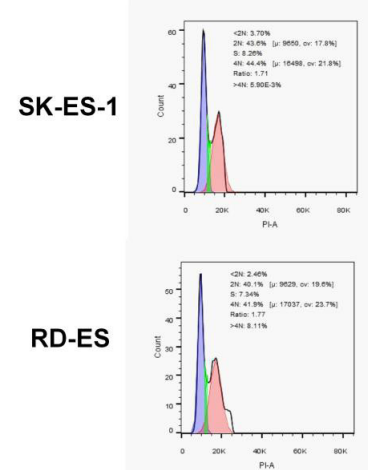

2.5
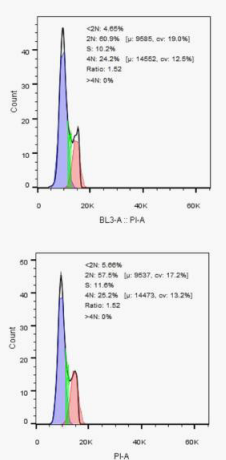

5
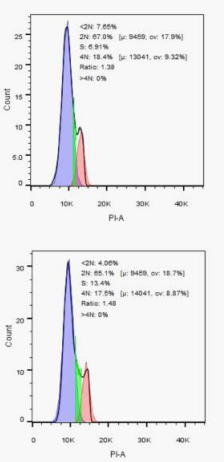

$10 \mathrm{mM}$
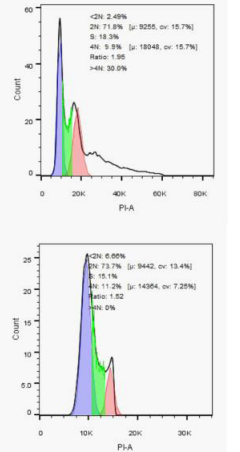

Fig. 2: Crocin blocked the cycle progression of human Ewing sarcoma cells (A) SK-ES-1 and (B) RD-ES in a dose-dependent manner Note: $(*)$ indicates $\mathbf{p}<0.05$ compared with the control group; $(* *)$ indicates $\mathbf{p}<0.01$ compared with the control group

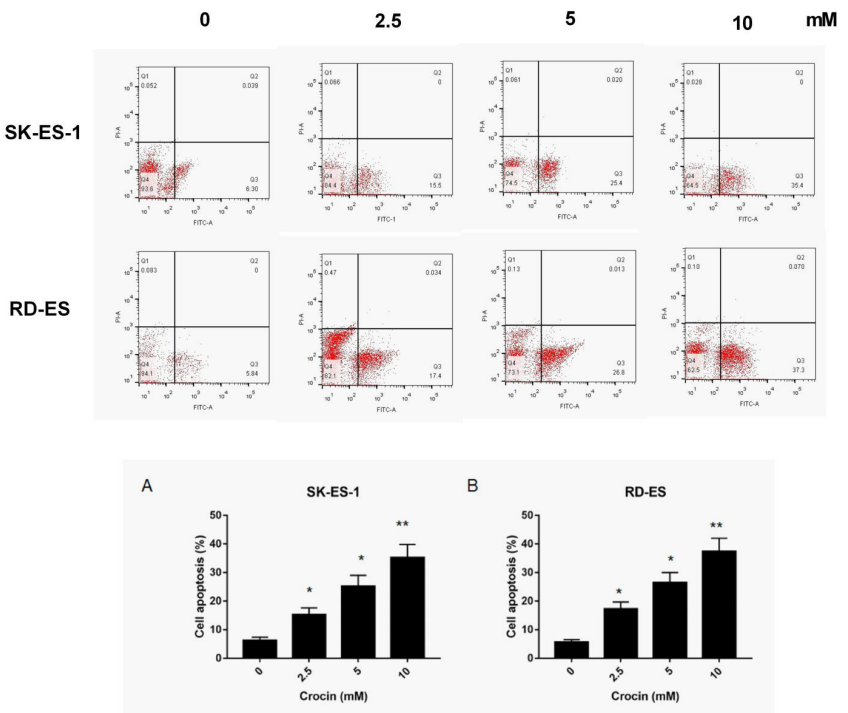

Fig. 3: Crocin could enhance the apoptosis of human Ewing sarcoma cells (A) SK-ES-1 and (B) RD-ES in a dose-dependent manner Note: (*) indicates that compared with the control group, the $\mathbf{p}<0.05 ;(* *)$ indicates that compared with the control group, the $\mathbf{p}<0.01$
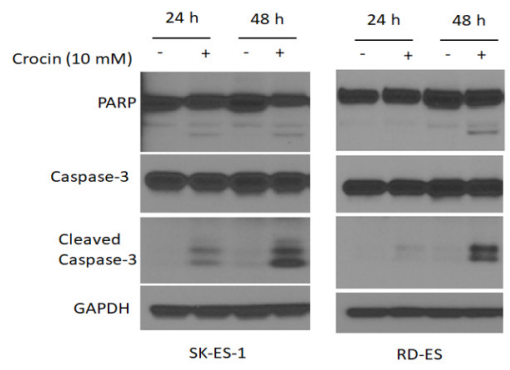

B
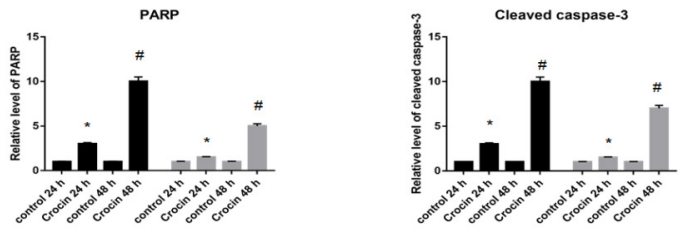

Fig. 4: Crocin could enhance the apoptosis of human Ewing sarcoma cells (A) SK-ES-1 and (B) RD-ES by affecting the degradation of caspase-3 and PARP

Note: (*) indicates compared with crocin $24 \mathrm{~h}$ group, the $\mathrm{p}<0.05$ and $\left(^{\#}\right)$ indicates compared with crocin $48 \mathrm{~h}$ group, the $\mathrm{p}<0.01$, ( -) SK-ES-1; ( $\mid$ ) RD-ES 

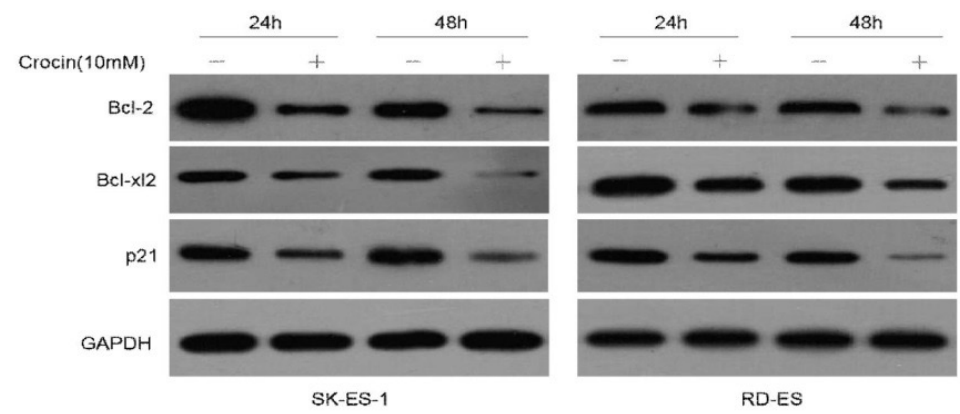

Bcl-2
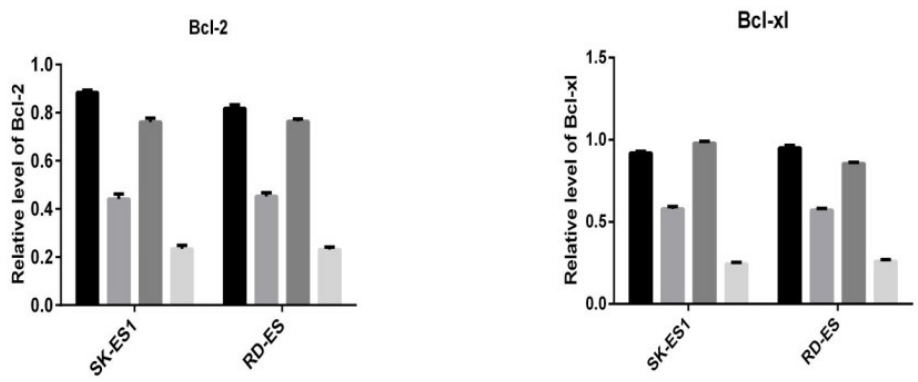

P21

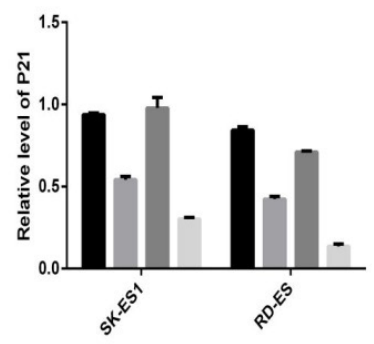

Fig. 5: Crocin could enhance the apoptosis of human Ewing sarcoma cells (A) SK-ES-1 and (B) RD-ES by regulating the expression of anti-apoptotic proteins

Note: (*) indicates that compared with crocin $24 \mathrm{~h}$ group, the $\mathrm{p}<0.05$ and $\left({ }^{\#}\right)$ indicates that compared with crocin $48 \mathrm{~h}$ group, the p< 0.01 , ( ש ) control 24 h; ( $\mid$ ) Crocin 24 h; ( $\square)$ Control 48 h; ( $\square)$ Crocin 48 h

A

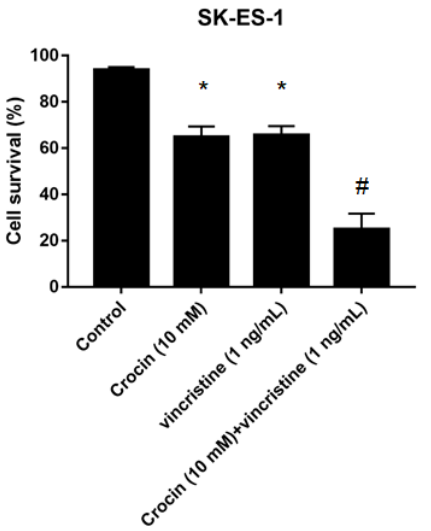

B

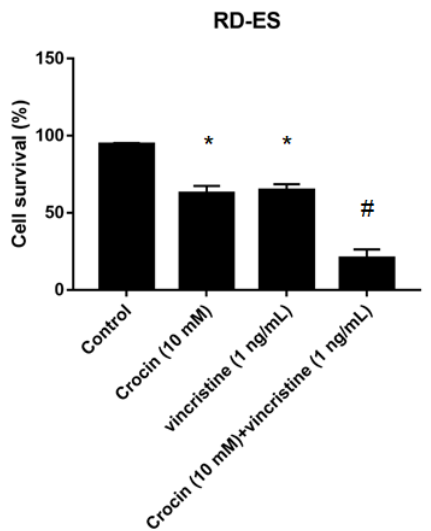

Fig. 6: Crocin could cooperate with vincristine to reduce the survival of human Ewing sarcoma cells (A) SK-ES-1 and (B) RD-ES Note: (*) indicates that compared with control group, the $\mathbf{p}<0.05$; $(*)$ indicates that compared with control group, the $p<0.01$ 

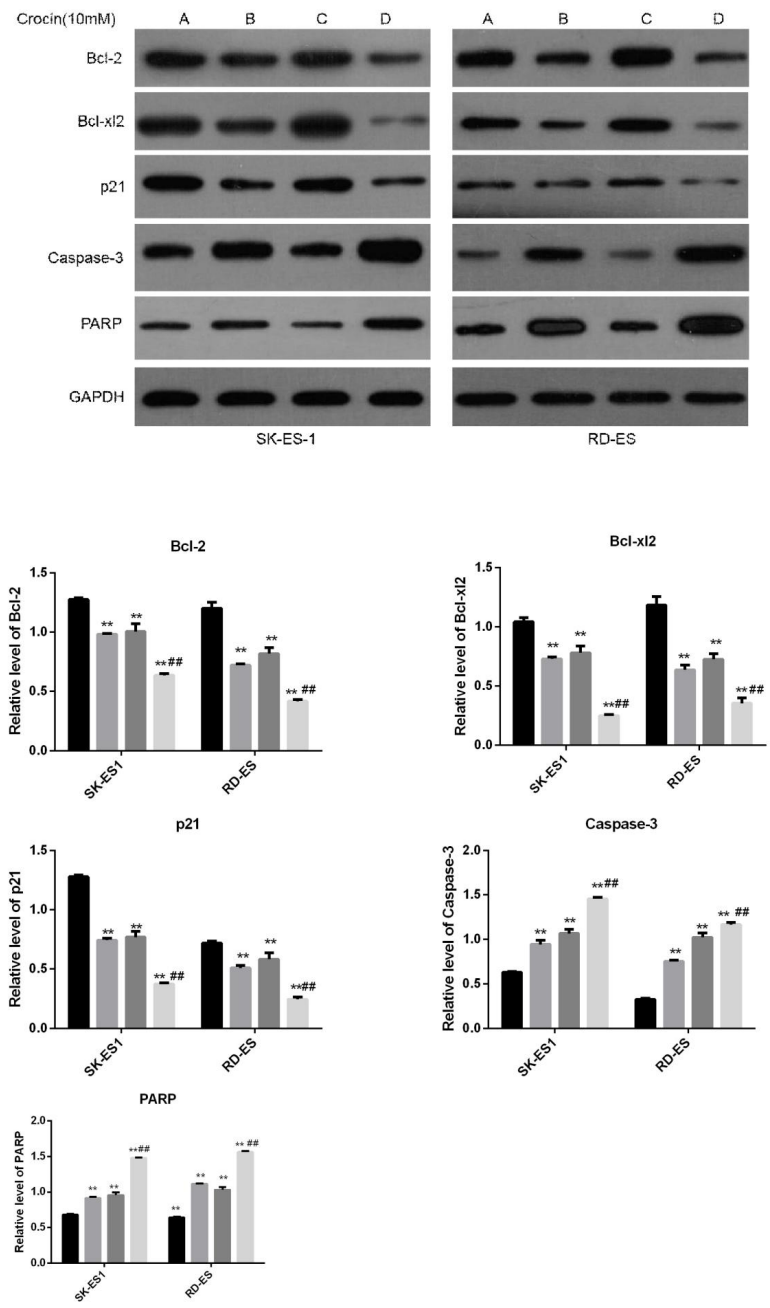

Fig. 7: Crocin could synergize with vincristine to promote apoptosis of human Ewing sarcoma cells

Note: $(*)$ indicates that compared with the control group, the $\mathbf{p}<0.05 ;\left(^{*}\right)$ indicates that compared with crocin and vincristine group, p<0.01, ( - ) Control group; ( $\mid$ ) Crocin group; ( - ) Vinvristine group; ( - ) Crocin+Vinvristine group

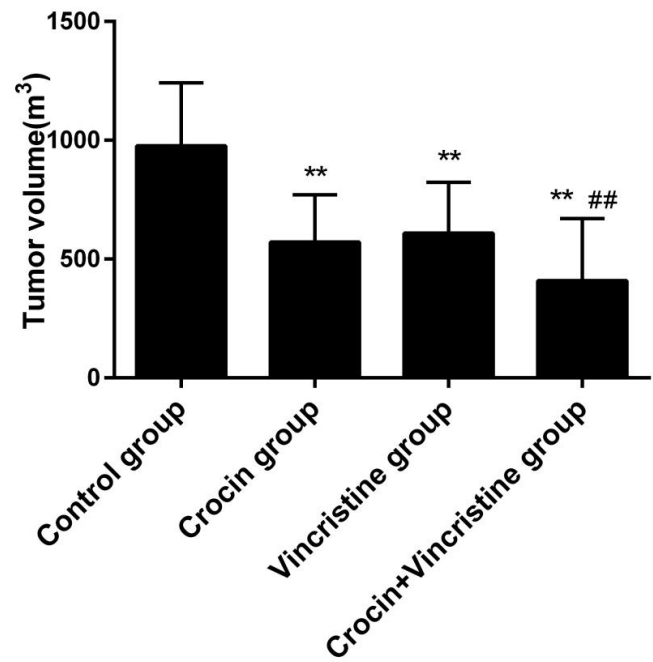

Fig. 8: Crocin could cooperate with vincristine to inhibit the growth of SK-ES-1 xenografts in nude mice Note: $(* *)$ indicates that compared to control group, the $\mathbf{p}<0.01 ;\left({ }^{\#}\right)$ indicates that compared with crocin and vincristine group, $\mathbf{p}<\mathbf{0 . 0 1}$ 


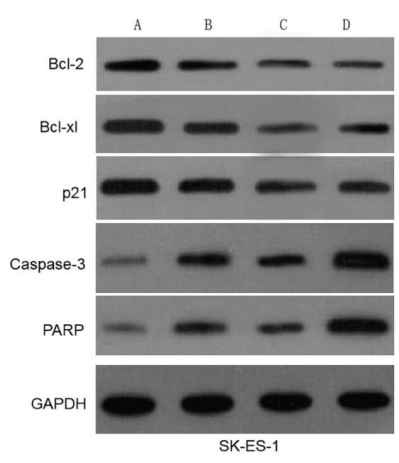

$\mathrm{Bcl}-\mathbf{x l}$

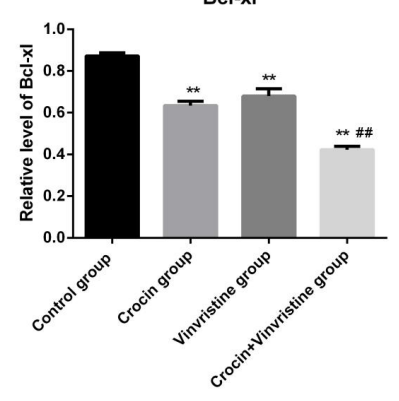

Caspase-3

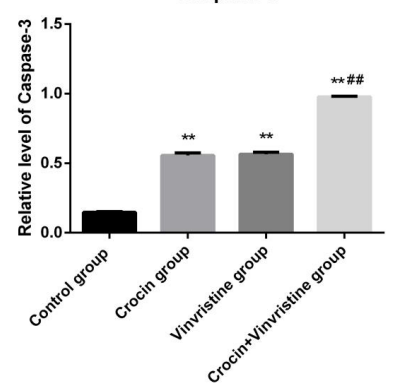

$\mathrm{Bcl}-2$

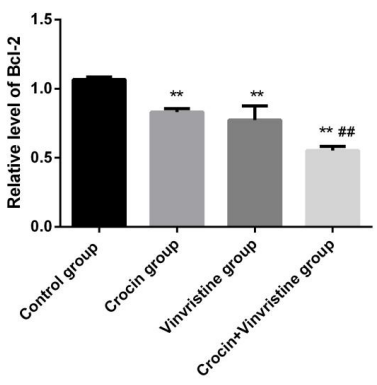

p21

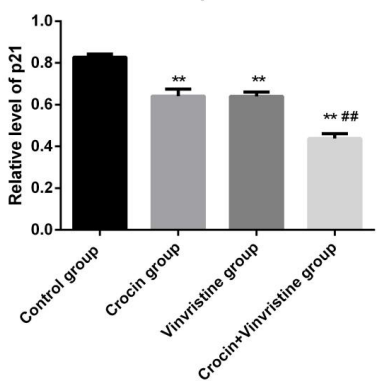

PARP

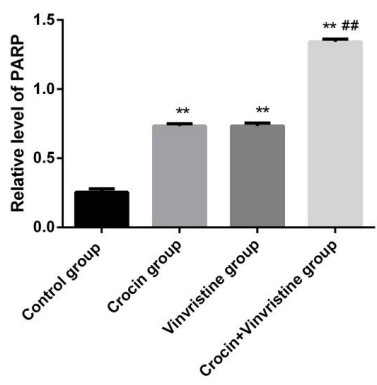

Fig. 9: Crocin could promote apoptosis of SK-ES-1 xenograft tumor cells in nude mice

Note: (*) indicates that compared to control group, the $\mathbf{p}<0.05$; $\left({ }^{*}\right)$ indicates that compared with crocin and vincristine group, the $\mathbf{p}<0.01$

Ewing sarcoma is the second most common primary pediatric bone tumor in children and young adults. This series of cancers is due to the balanced chromosomal translocation of the EWS gene and members of the Erythroblast Transformation Specific (ETS) gene family, the most common is being friend with Leukemia Virus Integration 1 (FLI1) ${ }^{[11,12]} .5$ y survival rates for children with local diseases ranged from 60 to $70 \%$ and for children with metastatic spread, these rates dropped to $30 \%$. Multimodal therapy is standard treatment, including systemic chemotherapy combined with radiotherapy or surgery ${ }^{[12]}$. But these therapies are not ideal for patients with distant metastasis and lack of a unified treatment standard, and the prognosis of patients is poor ${ }^{[13]}$. Therefore, it is necessary to find a new biological strategy for the treatment of this disease.
Recently, traditional Chinese medicine has been studied as a new source of anticancer drugs ${ }^{[14]}$. A great deal of effort has been devoted to finding drugs that can significantly induce apoptosis of cancer cells so far. The efficacy of minimizing side effects has become the main goal of discovering apoptotic inducers. Crocin is a diester formed by the disaccharide gentianase and crocetin dicarboxylic acid, which is the main active ingredient of Crocus sativus ${ }^{[15]}$. Studies have shown that crocin is an effective antioxidant ${ }^{[16]}$, therefore, it has been widely used to treat oxidative stress-related diseases and equipped with strong anticancer activity. It has been found that crocin could induce cancer cell death through p53-dependent and non-dependent mechanisms in colon cancer cells ${ }^{[17]}$. In addition, recent studies have shown that crocin inhibits the proliferation of human hepatocellular carcinoma cells by reducing 
human telomere reverse transcriptase ${ }^{[18]}$. Mostafavinia et al. ${ }^{[19]}$ showed that crocin could significantly inhibit the proliferation and apoptosis of breast cancer cells in a dose-and time-dependent manner and had a certain synergistic effect with radiotherapy. These studies prompted us to assume that crocin is a potential drug for the treatment of Ewing sarcoma.

Our results suggested that crocin could inhibit the growth of human Ewing sarcoma cells SK-ES-1 and RD-ES in a dose-dependent manner and increase the cell apoptosis. Mechanisms analysis showed that caspase-3 apoptotic pathway was up-regulated and anti-apoptotic pathway was down-regulated significantly. And crocin could increase the inhibitory effect of vincristine on human Ewing sarcoma.

Apoptosis or programmed cell death is a basic process necessary for tissue homeostasis and maintenance ${ }^{[20]}$. In this study, we first studied the inhibitory effect of crocin on Ewing sarcoma cells SK-ES-1 and RDES. The results showed that crocin could inhibit cell proliferation in a time- and dose-dependent manner. And the flow cytometry analysis showed that crocin could effectively induce cell apoptosis in a timeand dose-dependent manner and prevent cell cycle progression. These results suggested that crocin could inhibit the proliferation of Ewing sarcoma cells SKES-1 and RD-ES and promote cell apoptosis. At the same time, in vivo experiments showed that crocin could significantly inhibit the growth of xenograft tumor in nude mice, which was similar to $1 \mathrm{ng} / \mathrm{ml}$ of vincristine, while the volume of tumors in combination group was significantly lower than that in crocin group and vincristine group, suggesting that the combination of crocin and vincristine could inhibit the growth of Ewing sarcoma and both of them had synergistic effects.

Caspase induced apoptosis mainly through two pathways, mitochondrial (endogenous) pathway and death receptor (exogenous) pathway ${ }^{[21]}$. Mitochondrial pathways usually involve the induction of mitochondrial permeability transition and subsequent assembly of cytochrome c, Apoptotic Protease Activating Factor 1 (Apaf-1) and procaspase-9 into apoptotic bodies in the cytoplasm, leading to caspase- 9 activation and subsequent activation of caspase effects, such as caspase-3. The death receptor pathway involved the binding of death receptors to cell membranes and the recruitment of binding proteins Fas-Associated Death Domain (FADD) and procaspase- 8 to form a complex called death-inducing signal complex. Active caspase- 8 could activate caspase- 3 directly. In addition, effective apoptotic signaling usually required crosstalk between two pathways. Bid was an only proapoptotic member of Bcl-2 Homology domain 3 (BH3) of Bcl2 family, which was cut by caspase- 8 . The truncated Bid transported to mitochondria by activating apoptotic Bcl-2 family members BAX and BAK to permeate these organelles, leading to the release of cytochrome $\mathrm{c}$ and Second Mitochondria-Derived Activator of Caspase/ Direct Inhibitor of Apoptosis-Binding protein with LOw pI (SMAC/DIABLO). SMAC combined with apoptotic inhibitors to alleviate the complete activation of caspase-3. In this study, our results showed that $10 \mathrm{mM}$ crocin incubation can significantly increase PARP degradation and caspase-3 degradation and down-regulated the levels of anti-apoptotic proteins bcl-2, Bcl-xL and $\mathrm{p} 21$. There were consistent results in both cell level and in vivo experiments, suggesting that crocin may reduce cell survival by initiating the apoptotic process of cells.

In summary, crocin could inhibit the growth of human Ewing sarcoma either alone or in combination with vincristine, this effect could be achieved by activating caspase-related apoptotic pathway.

\section{Conflict of interests:}

The authors declared no conflicts of interest.

\section{REFERENCES}

1. Balamuth NJ, Womer RB. Ewing's sarcoma. Lancet Oncol 2010;11(2):184-92.

2. Rellinger EJ, Padmanabhan C, Qiao J, Appert A, Waterson AG, Lindsley CW, et al. M1327 induces apoptosis and sensitizes Ewing sarcoma cells to TNF-related apoptosis-inducing ligand. Biochem Biophys Res Commun 2017,491(2):463-8.

3. Zhao P, Alam MB, Lee SH, Kim YJ, Lee S, An H, et al. Spatholobus suberectus exhibits antidiabetic activity in vitro and in vivo through activation of AKT-AMPK pathway. Evid Based Complement Alternat Med 2017;2017.

4. Chen S, Zhao S, Wang X, Zhang L, Jiang E, Gu Y, et al. Crocin inhibits cell proliferation and enhances cisplatin and pemetrexed chemosensitivity in lung cancer cells. Transl Lung Cancer Res 2015;4(6):775-83.

5. Dehghan F, Hajiaghaalipour F, Yusof A, Muniandy S, Hosseini SA, Heydari S, et al. Saffron with resistance exercise improves diabetic parameters through the GLUT4/AMPK pathway in vitro and in vivo. Sci Rep 2016;6:25139.

6. Sun Y, Wang Z, Wang L, Wang LZ, Zang C, Sun LR. The effect and mechanisms of proliferative inhibition of crocin on human leukaemia jurkat cells. West Indian Med J 2015;64(5):473-9.

7. Jagadeeswaran R, Thirunavukkarasu C, Gunasekaran P, Ramamurty N, Sakthisekaran D. In vitro studies on the selective cytotoxic effect of crocetin and quercetin. Fitoterapia 2000;71(4):395-9.

8. Dhar A, Mehta S, Dhar G, Dhar K, Banerjee S, Veldhuizen PV, 
et al. Crocetin inhibits pancreatic cancer cell proliferation and tumor progression in a xenograft mouse model. Mol Cancer Ther 2009;8(2):315-23.

9. Chryssanthi DG, Dedes PG, Karamanos NK, Cordopatis P, Lamari FN. Crocetin inhibits invasiveness of MDAMB-231 breast cancer cells via downregulation of matrix metalloproteinases. Planta Med 2011;77(2):146-51.

10. Lu M, Gong X, Lu Y, Guo J, Wang C, Pan Y. Molecular cloning and functional characterization of a cell-permeable superoxide dismutase targeted to lung adenocarcinoma cells. Inhibition cell proliferation through the Akt/p27kip1 pathway. J Biol Chem 2006;281(19):13620-7.

11. Potratz J, Dirksen U, Jurgens H, Craft A. Ewing sarcoma: Clinical state-of-the-art. Pediatr Hematol Oncol 2012;29(1):111.

12. Gorlick R, Janeway K, Lessnick S, Randall RL, Marina N, COG Bone Tumor Committee. Children's oncology group's 2013 blueprint for research: Bone tumors. Pediatr Blood Cancer 2013;60(6):1009-15.

13. Ziqin L. Progress in the diagnosis and treatment of Ewing's sarcoma in children. Beijing Med J 2013;35(4):291-4.

14. Liu XP, He L, Zhang QP, Zeng XT, Liu SQ. Baicalein inhibits proliferation of myeloma U266 cells by downregulating IKZF1 and IKZF3. Med Sci Monit 2018;24:2809-17.

15. Konstantopoulos P, Doulamis IP, Tzani A, Korou ML, Agapitos E, Vlachos IS, et al. Metabolic effects of Crocus sativus and protective action against non-alcoholic fatty liver disease in diabetic rats. Biomed Rep 2017;6(5):513-8.

16. Jnaneshwari S, Hemshekhar M, Santhosh MS, Sunitha K, Thushara $\mathrm{R}$, Thirunavukkarasu $\mathrm{C}$, et al. Crocin, a dietary colorant, mitigates cyclophosphamide-induced organ toxicity by modulating antioxidant status and inflammatory cytokines. J Pharm Pharmacol 2013;65(4):604-14.
17. Li CY, Huang WF, Wang QL, Wang F, Cai E, Hu B, et al. Crocetin induces cytotoxicity in colon cancer cells via p53-independent mechanisms. Asian Pac J Cancer Prev 2012;13(8):3757-61.

18. Noureini SK, Wink M. Antiproliferative effects of crocin in HepG2 cells by telomerase inhibition and hTERT downregulation. Asian Pac J Cancer Prev 2012;13(5):2305-9.

19. Mostafavinia SE, Khorashadizadeh M, Hoshyar R. Antiproliferative and proapoptotic effects of crocin combined with hyperthermia on human breast cancer cells. DNA Cell Biol 2016;35(7):340-7.

20. Derakhshan A, Chen Z, van Waes C. Therapeutic small molecules target inhibitor of apoptosis proteins in cancers with deregulation of extrinsic and intrinsic cell death pathways. Clin Cancer Res 2017;23(6):1379-87.

21. Liu G, Zou H, Luo T, Long M, Bian J, Liu X, et al. Caspasedependent and caspase-independent pathways are involved in cadmium-induced apoptosis in primary rat proximal tubular cell culture. Plos One 2016;11(11):e0166823.

This is an open access article distributed under the terms of the Creative Commons Attribution-NonCommercial-ShareAlike 3.0 License, which allows others to remix, tweak, and build upon the work non-commercially, as long as the author is credited and the new creations are licensed under the identical terms

This article was originally published in a special issue, "Novel Therapeutic Approaches in Biomedicine and Pharmaceutical Sciences" Indian J Pharm Sci 2021:83(6) Spl Issue "38-47" 